\title{
Mitochondrial fission and fusion dynamics: the long and short of it
}

\author{
SB Berman ${ }^{1,2}$, FJ Pineda ${ }^{3,4}$ and JM Hardwick ${ }^{\star 2,3}$
}

Maintenance of functional mitochondria requires fusion and fission of these dynamic organelles. The proteins that regulate mitochondrial dynamics are now associated with a broad range of cellular functions. Mitochondrial fission and fusion are often viewed as a finely tuned balance within cells, yet an integrated and quantitative understanding of how these processes interact with each other and with other mitochondrial and cellular processes is not well formulated. Direct visual observation of mitochondrial fission and fusion events, together with computational approaches promise to provide new insight.

Cell Death and Differentiation (2008) 15, 1147-1152; doi:10.1038/cdd.2008.57; published online 25 April 2008

This article honors Richard Lockshin, whose vision saw so deeply and so clearly into the mechanisms of what he coined 'programmed cell death' even without all the technological advances in microscopy afforded to the work described here.

\section{Mitochondrial Dynamics}

Importance of mitochondrial fission and fusion. Mitochondrial fission and fusion are known to play roles in maintaining the (1) integrity of mitochondria, (2) electrical and biochemical connectivity, (3) turnover of mitochondria, and (4) segregation and protection of mitochondrial DNA (mtDNA). ${ }^{1-3}$ Moreover, the fission and fusion machinery have been implicated in programmed cell death pathways. The early fragmentation of mitochondria after an apoptotic stimulus is mediated in part by the mitochondrial fission proteins Drp1/ DLP1/Dnm1 and Fis1, and blocking the actions of Drp1 with a dominant negative mutant inhibits the fragmentation of mitochondria, and in some cases also prevents the loss of mitochondrial membrane potential and release of cytochrome $c^{4-7}$ In addition, it was reported that the mitochondrial inner membrane fusion protein Opa1 is intimately involved in cristae remodeling and protection from cytochrome $c$ release, ${ }^{8,9}$ suggesting that the fusion mechanisms are also important in normal mitochondrial cristae maintenance. For reasons that remain unclear, the maintenance of mtDNA cannot take place without mitochondrial fusion, ${ }^{10}$ and fusion of mitochondria has been directly implicated in preventing the accumulation of damaged mtDNA. ${ }^{11,12}$
In the special case of neurons, mitochondrial dynamics appear to play an even more critical role, perhaps because neurons have unusually long processes, electrical properties, complex energy requirements and synaptic functions such as neurotransmitter release. For example, the mitochondrial fission machinery is intimately involved in the formation and function of synapses in dendritic spines and axons. ${ }^{13,14} \mathrm{~A}$ powerful tool for revealing the importance of Drp1 (dynaminrelated protein) in these processes is a dominant-negative mutant of the fission protein Drp1, such as Drp1 ${ }^{\mathrm{K} 38 \mathrm{~A}}$ with impaired GTPase activity. Exogenously expressed, Drp1 ${ }^{\mathrm{K} 38 \mathrm{~A}}$ causes a loss of mitochondria from dendritic spines and a reduction in synapse number and size, whereas enhanced mitochondrial fission increases synapse formation. ${ }^{13,14}$ Depletion of endogenous Drp1 has also been shown to prevent mitochondria from distributing to synapses and to cause synaptic dysfunction ${ }^{15,14}$ consistent with a recently described human mutation in Drp1/DLP1 associated with severe abnormal brain development. ${ }^{16}$ Similarly, mutations in mitochondrial fusion factors cause a number of different human neuropathies ${ }^{17-19}$ and defective mitochondrial dynamics are increasingly implicated in many other neurodegenerative diseases. ${ }^{20,21}$

\section{What does Mitochondrial Morphology Tell about Fusion and Fission?}

The fluidity and dynamic properties of intramitochondrial membranes, especially mitochondrial cristae, were first

\footnotetext{
${ }^{1}$ Pittsburgh Institute for Neurodegenerative Diseases and Department of Neurology, University of Pittsburgh, Pittsburgh, PA 15260, USA; ${ }^{2}$ Department of Neurology, Johns Hopkins School of Medicine, Baltimore, MD 21205, USA; ${ }^{3}$ W. Harry Feinstone Department of Molecular Microbiology and Immunology, Johns Hopkins University Bloomberg School of Public Health, Baltimore, MD 21205, USA and ${ }^{4}$ Department of Biostatistics, Johns Hopkins University Bloomberg School of Public Health, Baltimore, MD 21205, USA

*Correspondence: JM Hardwick, Department of Molecular Microbiology and Immunology, Johns Hopkins University, 615 N Wolfe St., MMI E5140, Baltimore, MD 21205, USA. Tel: + 410955 2716; Fax: + 410955 0105;

E-mail: mhardwic@jhsph.edu

Keywords: mitochondria; fusion; fission; Drp1; Fis1; Fzo1

Abbreviations: Bcl-2, B-cell lymphoma/leukemia protein 2; CED-9, cell death-defective Bcl-2 homolog of worms; Drp1 ${ }^{*}$, dynamin-related protein 1; FRAP**, fluorescence recovery after photobleaching; Fzo1, fuzzy onion protein 1 of mammals/yeast (Drosophila fuzzy onions); GFP, green fluorescent protein; Mfn1, mitofusin protein 1 (mammalian Fzo1); mt, mitochondrial (e.g. mtDNA, mtGFP, mtRFP); RFP, red fluorescent protein; TEM ${ }^{\star *}$, transmission electron microscopy

Received 31.1.08; revised 20.3.08; accepted 21.3.08; Edited by G Melino; published online 25.4.08
} 
suggested by transmission electron microscopy (TEM), and this technique has continued to reveal new insights into cristae remodeling. ${ }^{22}$ In addition, even before more advanced techniques, TEM and phase contrast microscopy revealed multiple mitochondrial morphologies, and dynamic changes in morphology and structure, suggesting direct division and fusion of individual mitochondrial organelles (reviewed in Bereiter-Hahn and Voth ${ }^{23}$ ). Recent advancements in live cell imaging and high throughput strategies have rapidly advanced our appreciation for the importance of mitochondrial dynamics in cell death and survival; yet, relatively little is known about the molecular details, and much less is known about how these dynamic processes interface with mitochondrial energetics.

The $f z o$ (fuzzy onions) gene is a fusion mediator that was first identified in Drosophila where loss-of-function mutations in $f z O$ were shown to be responsible for defects in spermatogenesis. ${ }^{24}$ The phenotype of these mutants is a failure of spermatid mitochondria to merge into two supersized organelles to form the Nebenkern that appears onion-like in cross section. The connected mitochondrial morphology of $f z o$ mutant fly spermatids implied a defect in mitochondrial fusion, which was born out by elegant genetic experiments in yeast. Interestingly, loss-of-function mutations in the Drosophila homolog of human parkin, which causes autosomal recessive juvenile Parkinson disease when mutated, results in defective mitochondrial morphogenesis in the fly such that only one of the two giant mitochondria from the Nebenkern unfolds, leading to additional abnormal morphologies and condensation. ${ }^{25}$

Genetic studies in the budding yeast Saccharomyces cerevisiae have identified over 20 genes that are required for normal mitochondrial morphology (Figure 1), and orthologs of some of these factors were subsequently identified in mammals (reviewed in Okamoto and Shaw ${ }^{1}$ and Hoppins ${ }^{26}$ ). The conclusion that yeast Dnm1 (ortholog of mammalian
Drp1) reduces the length of mitochondria by mediating organelle fission, rather than inhibiting fusion or other mechanism, is based on compelling genetic experiments using morphology-based readouts. Conversely, yeast Fzo1 (fuzzy onion-1), ortholog of mammalian mitofusin (Mfn1 and Mfn2), increases the connectivity of mitochondria by mediating fusion, rather than inhibiting fission. However, all of these conclusions were based only on indirect evidence rather than by directly enumerating fusion and fission events in live cells. The relatively recent advent of experimental methods that permit the direct observation of individual mitochondrial fission and fusion events are enabling more detailed analyses but are also introducing additional complications in quantifying and interpreting the data as explored in the following sections.

How do we know that Fzo/Mfn is a fusion protein rather than a fission inhibitor? A genetic approach in yeast provided indirect, yet, unambiguous evidence that yeast Fzo1 is a fusion protein. ${ }^{27}$ During mating and zygote formation, the mitochondria from one haploid parent fuses to that of the other mating type parent. Therefore, to establish that yeast Fzo1 mediates fusion rather than suppressing fission, mitochondria of one haploid parent were stained with MitoTracker, while the other parent expressed mitochondrially targeted GFP (mitoGFP). One or both parents also carried a deletion or a temperature-sensitive mutation in the FZO1 gene. At the permissive temperature, the merge of green and red mitochondria occurred normally, indicating that the mitochondria from one cell fused with that of the second cell. However, at the elevated temperature, where the mutant Fzo1 protein is non-functional or when the FZO1 gene was deleted, which causes short punctate mitochondrial morphology, little or no merge of red and green mitochondria occurred following mating. Therefore, Fzo1 was required to facilitate mitochondrial fusion, and the presence of Fzo1 on one mitochondrion was not sufficient for it to fuse to a Fzo1-deficient mitochondrion.

Yeast proteins required for normal mitochondrial morphology

\begin{tabular}{lcll} 
Protein & human homolog & & \multicolumn{1}{c}{ Possible role/localization } \\
\cline { 2 - 3 } Caf4 & - & & Mdv1-like, outer membrane fission, interacts with Dnm1, Mdv1 and Fis1 \\
Cym1 & - & & Pitrilysin-like, intermembrane space metalloprotease \\
Dnm1 & Drp1/DLP1 & & Dynamin-related GTPase, outer membrane fission \\
Fis1 & Fis1 & & Outer membrane-anchored fission protein \\
Fzo1 & Mfn1,2 & & Integral membrane protein with N-terminal GTPase \\
Gem1 & - & Mitochondrial GTPase, EF-hand protein \\
Htd2 & - & 3-hydroxyacyl-thioester dehydratase \\
Mam3 & - & Hemolysin-like \\
Mdm20 & - & F-terminal acetyltransferase \\
Mdm30 & - & Inner membrane \\
Mdm31 & - & Similar to MDM31, inner membrane \\
Mdm32 & - & May promote mitochondrial inner membrane fission \\
Mdm33/She9 & - & Outer membrane \\
Mdm34/Mmm2 & - & Intermembrane space \\
Mdm35 & - & Unknown \\
Mdm36 & - & Rhomboid-like mitochondrial intermembrane protease \\
Mdm37/Pcp1 & - & Mitochondrial inner membrane \\
Mdm38/Mkh1 & - & Unknown \\
Mdm39/Get1 & - & WD repeat protein, complexes with Dnm1 and Fis1 \\
Mdv1 & - & - & F-box protein \\
Mfb1 & Opa1 & Dynamin-like GTPase, cristae morphology, complexes with Fzo1 and Ugo1 \\
Mgm1 & PREL1 & Binds Fzo1 and Mgm1 \\
Ugo1 & Intermembrane space, regulates Mgm1 \\
Ups1 & & &
\end{tabular}

Figure 1 Yeast proteins required for normal mitochondrial morphology through their direct or indirect roles; for more information and citations, see Saccharomyces Genome Database at http://www.yeastgenome.org/ 
Furthermore, deletion of both FZO1 and the mitochondrial fission gene DNM1/Drp1 restored normal tubular mitochondrial morphology, but failed to restore mitochondrial fusion upon mating of these double knockouts. ${ }^{28}$ Thus, the restoration of tubular morphology in the yeast double knockout presumably occurred through elongation of mitochondrial organelles rather than fusion. Subsequently, the mammalian counterparts, Mfn1 and Mfn2, were reported to mediate mitochondrial fusion, though this conclusion was also based on short mitochondrial morphology when the function of Mfn1/Mfn2 was impaired. ${ }^{29,30}$

While these studies in yeast are compelling, it remains possible that the effects of mutation or deletion of FZO1 on mitochondrial morphology are merely downstream consequences of a direct action on an entirely distinct process, such as energetics, ion flux, or other. However, additional evidence argues that Fzo/Mfn proteins are direct mediators of mitochondrial fusion. In particular, Fzo/Mfn proteins share significant amino-acid sequence similarity to the large dynamin GTPases known to be involved in membrane and vesicle fission. ${ }^{31}$ Furthermore, yeast Fzo1 protein was shown to be embedded in the mitochondrial outer membrane. ${ }^{10,27}$ More importantly, an in vitro assay was developed to demonstrate Fzo1-dependent fusion of mitochondria, thereby solidifying the role of Fzo/Mfn in membrane fusion. ${ }^{32}$ In these studies, yeast mitochondria labeled with mitochondrially targeted DsRed fluorescent protein were mixed with yeast mitochondria labeled with mitoGFP. ${ }^{32}$ Directly quantified mitochondrial fusion events were evaluated under different conditions, and a direct role for Fzo1 was confirmed. In addition, physiologic conditions, such as mitochondrial membrane potential and GTP, were required for mitochondrial fusion in vitro, and inner membrane fusion could be distinguished from outer membrane fusion.

Changes in morphology are generally taken as evidence for a change in the balance between fission and fusion. ${ }^{33-35}$ The use of morphology as a readout, is largely due to the difficulty of directly quantifying fission and fusion processes.

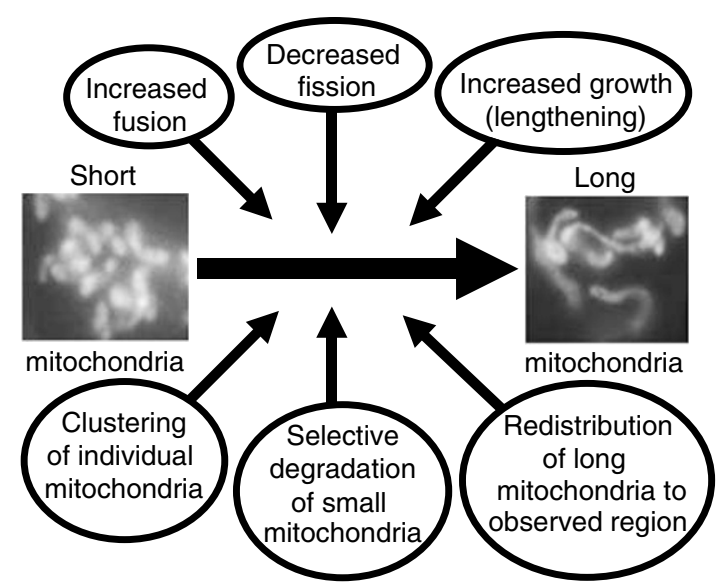

Figure 2 When the equilibrium between mitochondrial fission and fusion is reset to yield longer, more connected mitochondrial morphology, detected here in mammalian cells with mitoGFP, there are a number of potential mechanisms, or combinations of mechanisms to explain this altered morphology
Unfortunately, morphologic changes are not particularly revealing about biochemical processes. Other than a defective mitochondrial fission, fusion or growth extension and the countless potential sources of experimental bias, elongated/ connected mitochondrial morphology could be due to changes in organelle distribution (or plane of focus), organelle stretching, or selective degradation of short mitochondria (Figure 2).

\section{Indirect methods for assessing mitochondrial fission and fusion in mammalian cells}

Cell-cell fusion. Modeled after the strategies employed in yeast, experimentally induced cell-cell fusion of two cell populations, labeled differentially with mitochondrially targeted RFP (mitoRPF) or mitoGFP, has been applied to monitor the mixing of mammalian mitochondrial matrices. $^{36,37}$ These studies verified the conservation of fission and fusion machinery between yeast and mammals. As in yeast, mammalian mitochondrial fusion is also dependent on mitochondrial membrane potential and is not dependent on mitochondrial respiration, though some discrepancies were reported. ${ }^{37}$

Fluorescence recovery after photobleaching (FRAP). To avoid potential artifacts from membrane-altering agents required to experimentally induce cell-cell fusion, new technologies were developed to more closely mimic in vivo conditions, in which physiologic manipulations and disease models can be studied. FRAP has been increasingly utilized to assess mitochondrial connectivity. Photobleached subcellular regions are monitored for refilling of 'bleached' areas with mitochondrially targeted fluorescent molecules from neighboring fluorescent (unbleached) areas to provide evidence that bleached mitochondria are physically connected to structures outside the bleached area. For example, this approach was used to show that the $C$. elegans $\mathrm{Bcl}-2$ homolog, CED-9, promotes mitochondrial fusion in HeLa cells. ${ }^{38}$

Photoactivatable mitoGFP. The ability to monitor fission and fusion of individual mitochondria was facilitated by photoactivatable mitoGFP. Photoactivatable GFP is a variant of Aequora victoria GFP, in which a single aminoacid substitution renders a stable, 100-fold increase in green fluorescence after laser activation. ${ }^{39}$ Karbowski et al. ${ }^{40}$ fused photoactivatable GFP to a mitochondrial targeting sequence. When coexpressed with a constitutively fluorescent marker, the interaction of activated and constitutively fluorescent mitochondrial populations can be monitored. For example, the loss of photoactivated fluorescence in a small region of mitochondria has been utilized as a measure of mitochondrial fusion, assuming that this loss of photoactivated fluorescence represents diffusion of fluorescence into a neighboring (presumably now fused) mitochondrion. This methodology was used to support a direct role for mitochondrial fusion by the $\mathrm{Bcl}-2$ family proteins Bax and Bak. ${ }^{41}$ Photoactivated plus potentiometric mitochondrial fluorescence led to the interesting observation that one of the two daughter mitochondria produced by a fission event undergoes transient membrane depolarisation, a possible marker for mitochondrial deletion. ${ }^{42}$ 
Mitochondrial visitation without fusion

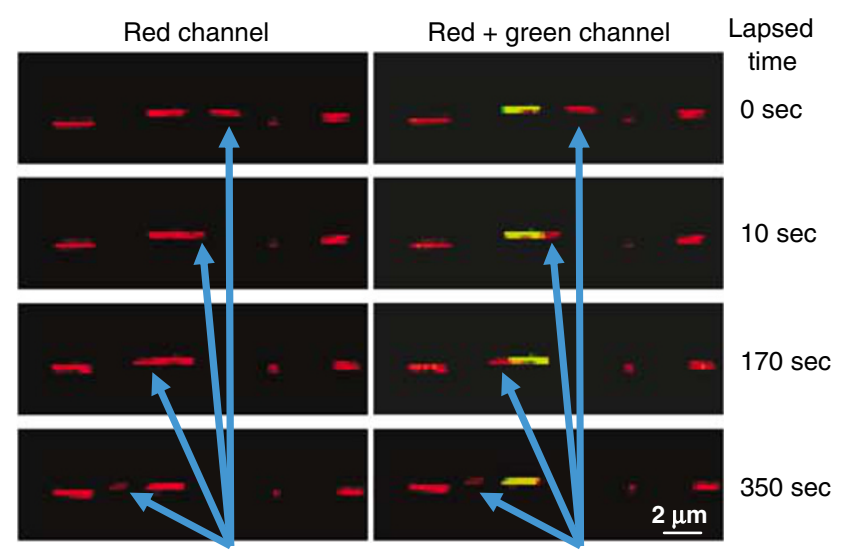

Mito-RFP + photoactivatable mito-GFP

Figure 3 Monitoring mitochondrial movement. Time-lapse imaging was performed on a 14-day culture of primary rat cortical neurons cotransfected with mitochondrially targeted RFP and photoactivatable GFP; one mitochondrion was photoactivated (yellow). Blue arrows track the movement of an RFP-labeled mitochondrion past the photoactivated mitochondrion. Under RFP fluorescence detection alone, one cannot distinguish the monitored (red) and the photoactivated mitochondrion when both are juxtaposed, and it cannot be determined whether these mitochondria fuse. Using photoactivatable GFP, it can be concluded that these two mitochondria fail to fuse their matrices, even though they spend several minutes next to each other. Time 0 is at $8 \mathrm{~min}$ and $40 \mathrm{~s}$ from the start of a $15 \mathrm{~min}$ observation period

\section{Towards a more Quantitative Understanding of Mitochondrial Fission/Fusion Dyanamics}

Mitochondrial fusion can be quantified using the above methods, when there is a relatively stationary mitochondrial population. However, recovery from photobleaching and dispersal of photoactivated fluorescence intensities will obviously be compromised where populations of mitochondria are more mobile. For example, rapid transport up and down neuronal axons and dendrites can move mitochondria considerable distances in seconds/minutes. Thus, increased 'recovery of fluorescence' after photobleaching could indicate organelle connectivity due to increased fusion, or it could reflect the appearance of a new mitochondrion moving into the bleached area; and conversely, 'loss of fluorescence' could results from diffusion into another mitochondrion upon fusion, or a fluorescently activated mitochondrion was transported out of the field. Consequently, these methods will be less useful for assessing mitochondrial fusion in healthy neuronal processes because of the motility and rapid transport of mitochondria along processes.

Direct visualization of photoactivated mitochondrial fluorescence into an adjacent mitochondrion has been described. ${ }^{40}$ Using this method, together with improved image analyses, it will be possible to directly visualize, quantify, characterize, and manipulate mitochondrial fission/fusion dynamics in live neurons in real time. We have observed that selective photoactivation allows distinctions to be made between elongated mitochondria and structures that only appear continuous, but are instead clusters of individual mitochondria, or mitochondria that are being transported past each other (Figure 3). We believe that this methodology will

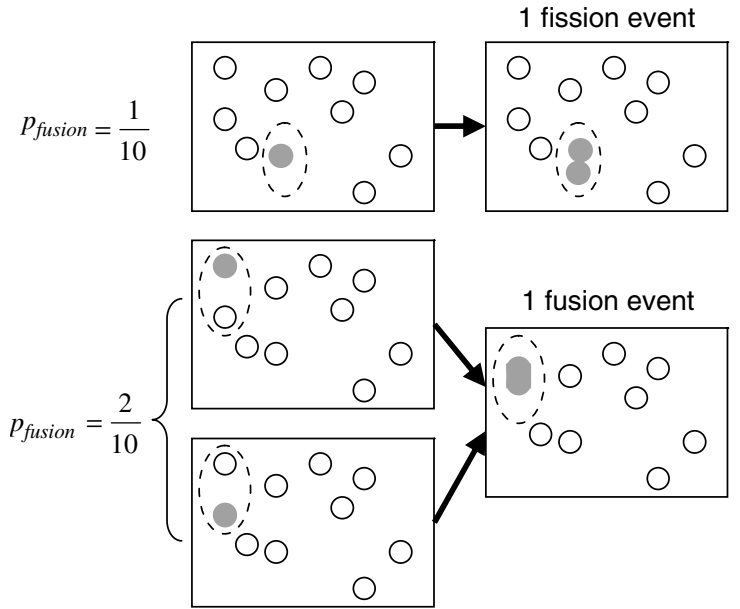

Figure 4 Quantifying mitochondrial fission and fusion. Consider a volume containing 10 mitochondria in which, on average, there is one fission event and one fusion event per a fixed time interval. If one of the 10 mitochondria is randomly selected for photoactivation, then one is twice as likely to activate a mitochondrion that will undergo a fusion event $\left(p_{\text {fussion }}=2 / 10\right)$ during the observation period, than one is likely to select a mitochondrion that will undergo a fission event $\left(p_{\text {fussion }}=1 / 10\right.$ ). Thus, one will observe twice as many fusion events as fission events, even though the number of fission events equals the number of fusion events

ultimately allow direct quantification of mitochondrial fission, fusion, and transport in living cells to better characterize physiologic and disease parameters.

\section{Future Directions}

A systems biology approach for mitochondrial fission and fusion. Although photoactivatable mitoGFP allows one to characterize individual fission and fusion events in a population of mitochondria, there still remains the task of interpreting the data and connecting these measurements to falsifiable hypotheses about morphology. Measured fission and fusion rates are the observable consequences of complex interactions between individual mitochondria. At a very coarse level, observed fusion rates must certainly depend on how often mitochondria encounter each other, and on the likelihood that two mitochondria will fuse when they do encounter each other. Thus, observed rates of fission and fusion events will depend indirectly on parameters such as the number of mitochondria per cell and the mean velocity of the mitochondrial population. How can we tease these parameters apart to reach an understanding of the underlying fission and fusion processes? We believe that these questions will be answered by developing and testing specific mathematical models of mitochondrial fission, fusion, and morphology. Careful modeling will be required to transform experimental observations into quantitative statements about the balance between fission, fusion, and its impact on morphology.

A simple, but important, question highlights the need for careful modeling: how does one define (in a rigorous sense) the balance between fission and fusion? Experimentally, one can measure the ratio of fission and fusion events in GFP photoactivated mitochondria. How are these observed rates 
connected to fission and fusion rates in the population? In the case of fission, if an observed fraction of GFP-activated mitochondria are observed to undergo fission within a specific time interval, the expected number of fission events in the population is obtained by multiplying the observed fission rate by the number of mitochondria in the volume. In the case of fusion, however, the situation is more subtle, as the same procedure (multiplying the observed fusion rate of photoactivated mitochondria by the number of mitochondria in the volume) will overestimate the number of fusion events in the volume by exactly a factor of two (Figure 4).

It becomes even more complex when one considers incorporating analysis of mitochondrial dynamics into steady-state physiological conditions. In other words, when subtle changes in mitochondrial fission or fusion occur inside cells, how do they affect and integrate with the balance of physiological parameters such as mitochondrial growth, biogenesis, degradation, metabolic function, and so on? If unregulated, one could predict that even small increases in the rates of mitochondrial fission would result in the eventual severe fragmentation of all mitochondria within a cell, but in normal in vivo steady-state conditions this does not occur, and changes in mitochondrial dynamics obviously integrate with the other mitochondrial processes. How is the balance maintained? A system-level understanding of how subtle regulation of mitochondrial fission and fusion interacts with the larger mitochondrial and cellular environment will clearly be critical for understanding the role of mitochondrial fission and fusion in physiologic cell function and dysfunction.

We often take for granted that there is a coordinated balance of mitochondrial fission and fusion that underlies not only morphology but also extends to involvement in cell death mechanisms and cell maintenance, and in neurons, to synaptic formation and function, and perhaps to neurodegeneration. However, tools to provide quantitative characterization of the complicated interrelationships between mitochondrial fission and fusion, and between mitochondrial dynamics and other cellular processes, have yet to be developed. As these are developed, we should be able to better incorporate scientific findings into physiologic implications for cell maintenance and disease.

Acknowledgements. We thank Kelly $\mathrm{M}$ Leach for the microscopy images in Figure 2. The work described here was supported by NIH Grant RO1-GM077875 (JMH).

1. Okamoto K, Shaw JM. Mitochondrial morphology and dynamics in yeast and multicellular eukaryotes. Annu Rev Genet 2005; 39: 503-536.

2. Hollenbeck PJ, Saxton WM. The axonal transport of mitochondria. J Cell Sci 2005; 118 5411-5419.

3. Westermann B. Merging mitochondria matters: cellular role and molecular machinery of mitochondrial fusion. EMBO Rep 2002; 3: 527-531.

4. Frank S, Gaume B, Bergmann-Leitner ES, Leitner WW, Robert EG, Catez F et al. The role of dynamin-related protein 1, a mediator of mitochondrial fission, in apoptosis. Dev Cell 2001; 1: 515-525.

5. Breckenridge DG, Stojanovic M, Marcellus RC, Shore GC. Caspase cleavage product of BAP31 induces mitochondrial fission through endoplasmic reticulum calcium signals, enhancing cytochrome c release to the cytosol. J Cell Biol 2003; 160: 1115-1127.

6. Lee YJ, Jeong SY, Karbowski M, Smith CL, Youle RJ. Roles of the mammalian mitochondrial fission and fusion mediators Fis1, Drp1, and Opa1 in apoptosis. Mol Biol Cell 2004; 15: 5001-5011.
7. Cassidy-Stone A, Chipuk JE, Ingerman E, Song C, Yoo C, Kuwana T et al. Chemical inhibition of the mitochondrial division dynamin reveals its role in Bax/Bak-dependent mitochondrial outer membrane permeabilization. Dev Cell 2008; 14: 193-204.

8. Arnoult D, Grodet A, Lee YJ, Estaquier J, Blackstone C. Release of OPA1 during apoptosis participates in the rapid and complete release of cytochrome $C$ and subsequent mitochondrial fragmentation. J Biol Chem 2005; 280: 35742-35750.

9. Frezza C, Cipolat S, Martins de Brito O, Micaroni M, Beznoussenko GV, Rudka T et al. OPA1 controls apoptotic cristae remodeling independently from mitochondrial fusion. Cell 2006; 126: 177-189.

10. Rapaport D, Brunner M, Neupert W, Westermann B. Fzo1p is a mitochondrial outer membrane protein essential for the biogenesis of functional mitochondria in Saccharomyces cerevisiae. J Biol Chem 1998; 273: 20150-20155.

11. Nakada $\mathrm{K}$, Inoue $\mathrm{K}$, Ono $\mathrm{T}$, Isobe $\mathrm{K}$, Ogura A, Goto $\mathrm{YI}$ et al. Inter-mitochondrial complementation: mitochondria-specific system preventing mice from expression of disease phenotypes by mutant mtDNA. Nat Med 2001; 7: 934-940.

12. Ono $\mathrm{T}$, Isobe $\mathrm{K}$, Nakada $\mathrm{K}$, Hayashi Jl. Human cells are protected from mitochondrial dysfunction by complementation of DNA products in fused mitochondria. Nat Genet 2001; 28: $272-275$

13. Li Z, Okamoto K, Hayashi $Y$, Sheng M. The importance of dendritic mitochondria in the morphogenesis and plasticity of spines and synapses. Cell 2004; 119: 873-887.

14. Li H, Chen Y, Jones AF, Sanger RH, Collis LP, Flannery R et al. Bcl-xL induces Drp1dependent synapse formation in cultured hippocampal neurons. Proc Natl Acad Sci USA 2008; 105: 2169-2174.

15. Verstreken P, Ly CV, Venken KJ, Koh TW, Zhou Y, Bellen HJ. Synaptic mitochondria are critical for mobilization of reserve pool vesicles at Drosophila neuromuscular junctions. Neuron 2005; 47: 365-378

16. Waterham HR, Koster J, van Roermund CW, Mooyer PA, Wanders RJ, Leonard JV. A lethal defect of mitochondrial and peroxisomal fission. N Engl J Med 2007; 356: $1736-1741$

17. Delettre C, Lenaers G, Griffoin JM, Gigarel N, Lorenzo C, Belenguer P et al. Nuclear gene OPA1, encoding a mitochondrial dynamin-related protein, is mutated in dominant optic atrophy. Nat Genet 2000; 26: 207-210.

18. Alexander C, Votruba M, Pesch UE, Thiselton DL, Mayer S, Moore A et al. OPA1, encoding a dynamin-related GTPase, is mutated in autosomal dominant optic atrophy linked to chromosome 3q28. Nat Genet 2000; 26: 211-215.

19. Zuchner S, Mersiyanova IV, Muglia M, Bissar-Tadmouri N, Rochelle J, Dadali EL et al. Mutations in the mitochondrial GTPase mitofusin 2 cause Charcot-Marie-Tooth neuropathy type 2A. Nat Genet 2004; 36: 449-451.

20. Chen $\mathrm{H}, \mathrm{McC}$ affery JM, Chan DC. Mitochondrial fusion protects against neurodegeneration in the cerebellum. Cell 2007; 130: 548-562.

21. Frank S. Dysregulation of mitochondrial fusion and fission: an emerging concept in neurodegeneration. Acta Neuropathol (Berl) 2006; 111: 93-100.

22. Mannella CA. The relevance of mitochondrial membrane topology to mitochondrial function. Biochim Biophys Acta 2006; 1762: 140-147.

23. Bereiter-Hahn J, Voth M. Dynamics of mitochondria in living cells: shape changes, dislocations, fusion, and fission of mitochondria. Microsc Res Tech 1994; 27 : 198-219.

24. Hales KG, Fuller MT. Developmentally regulated mitochondrial fusion mediated by a conserved, novel, predicted GTPase. Cell 1997; 90: 121-129.

25. Riparbelli MG, Callaini G. The Drosophila parkin homologue is required for normal mitochondrial dynamics during spermiogenesis. Dev Biol 2007; 303: 108-120.

26. Hoppins S, Lackner L, Nunnari J. The machines that divide and fuse mitochondria. Annu Rev Biochem 2007; 76: 751-780.

27. Hermann GJ, Thatcher JW, Mills JP, Hales KG, Fuller MT, Nunnari J et al. Mitochondrial fusion in yeast requires the transmembrane GTPase Fzo1p. J Cell Biol 1998; 143: 359-373.

28. Sesaki $H$, Jensen RE. Division versus fusion: Dnm1p and Fzo1p antagonistically regulate mitochondrial shape. J Cell Biol 1999; 147: 699-706.

29. Bach D, Pich S, Soriano FX, Vega N, Baumgartner B, Oriola J et al. Mitofusin-2 determines mitochondrial network architecture and mitochondrial metabolism. A novel regulatory mechanism altered in obesity. J Biol Chem 2003; 278: 17190-17197.

30. Eura $\mathrm{Y}$, Ishihara N, Yokota S, Mihara K. Two mitofusin proteins, mammalian homologues of FZO, with distinct functions are both required for mitochondrial fusion. $J$ Biochem (Tokyo) 2003; 134: 333-344.

31. Hinshaw JE. Dynamin and its role in membrane fission. Annu Rev Cell Dev Biol 2000; 16: 483-519.

32. Meeusen S, McCaffery JM, Nunnari J. Mitochondrial fusion intermediates revealed in vitro. Science 2004; 305: 1747-1752

33. Jendrach M, Pohl S, Voth M, Kowald A, Hammerstein P, Bereiter-Hahn J. Morpho-dynamic changes of mitochondria during ageing of human endothelial cells. Mech Ageing Dev 2005; 126: 813-821

34. Barsoum MJ, Yuan H, Gerencser AA, Liot G, Kushnareva Y, Gräber S et al. Nitric oxideinduced mitochondrial fission is regulated by dynamin-related GTPases in neurons. EMBO J 2006; 25: 3900-3911.

35. Yuan H, Gerencser AA, Liot G, Lipton SA, Ellisman M, Perkins GA et al. Mitochondrial fission is an upstream and required event for bax foci formation in response to nitric oxide in cortical neurons. Cell Death Differ 2007; 14: 462-471. 
36. Legros F, Lombes A, Frachon P, Rojo M. Mitochondrial fusion in human cells is efficient, requires the inner membrane potential, and is mediated by mitofusins. Mol Biol Cell 2002; 13: 4343-4354.

37. Malka F, Guillery O, Cifuentes-Diaz C, Guillou E, Belenguer $P$, Lombès A et al. Separate fusion of outer and inner mitochondrial membranes. EMBO Rep 2005; 6: 853-859.

38. Delivani P, Adrain C, Taylor RC, Duriez PJ, Martin SJ. Role for CED-9 and Egl-1 as regulators of mitochondrial fission and fusion dynamics. Mol Cell 2006; 21: 761-773.

39. Patterson GH, Lippincott-Schwartz J. A photoactivatable GFP for selective photolabeling of proteins and cells. Science 2002; 297: 1873-1877.
40. Karbowski M, Arnoult D, Chen $\mathrm{H}$, Chan DC, Smith CL, Youle RJ. Quantitation of mitochondrial dynamics by photolabeling of individual organelles shows that mitochondrial fusion is blocked during the Bax activation phase of apoptosis. J Cell Biol 2004; 164 : 493-499.

41. Karbowski M, Norris KL, Cleland MM, Jeong SY, Youle RJ. Role of Bax and Bak in mitochondrial morphogenesis. Nature 2006; 443: 658-662.

42. Twig G, Elorza A, Molina AJ, Mohamed H, Wikstrom JD, Walzer G et al. Fission and selective fusion govern mitochondrial segregation and elimination by autophagy. EMBO J 2008; 27: 433-446. 